Network Working Group

Request for Comments: 4657

Category: Informational
J. Ash, Ed. $\mathrm{AT} \& \mathrm{~T}$

J.L. Le Roux, Ed. France Telecom September 2006

\title{
Path Computation Element (PCE) Communication Protocol Generic Requirements
}

Status of This Memo

This memo provides information for the Internet community. It does not specify an Internet standard of any kind. Distribution of this memo is unlimited.

Copyright Notice

Copyright (C) The Internet Society (2006).

Abstract

The PCE model is described in the "PCE Architecture" document and facilitates path computation requests from Path Computation Clients (PCCs) to Path Computation Elements (PCEs). This document specifies generic requirements for a communication protocol between PCCs and PCEs, and also between PCEs where cooperation between PCEs is desirable. Subsequent documents will specify application-specific requirements for the PCE communication protocol.

Table of Contents

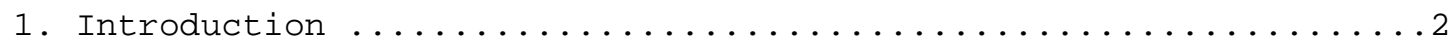

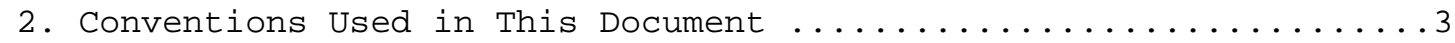

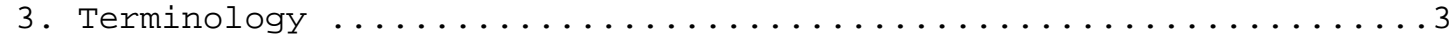

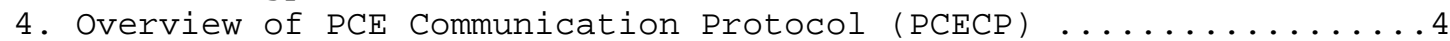

5. PCE Communication Protocol Generic Requirements .............5

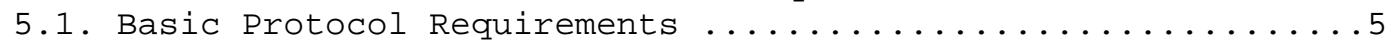

5.1.1. Commonality of PCC-PCE and PCE-PCE Communication ....5

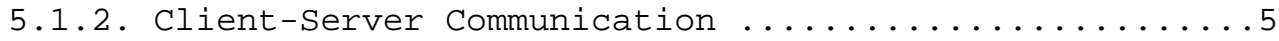

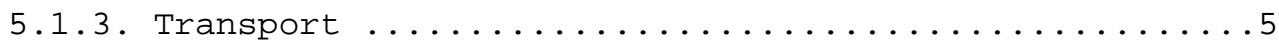

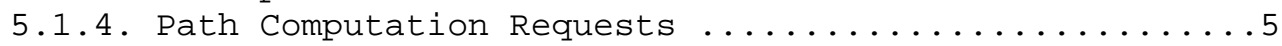

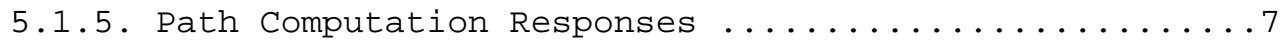

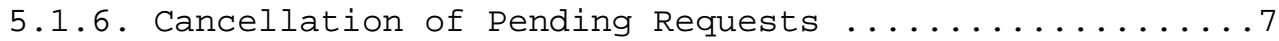

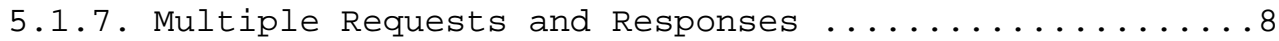

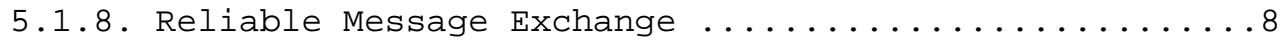

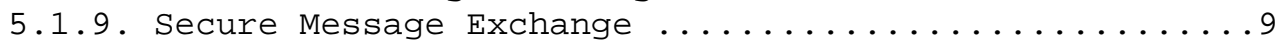




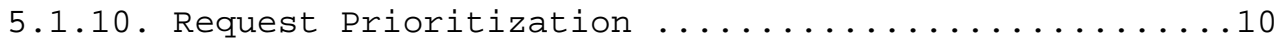

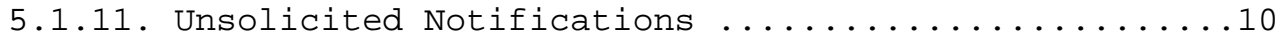

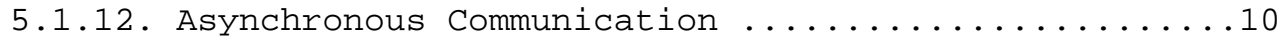

5.1.13. Communication Overhead Minimization ............10

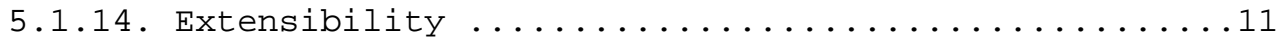

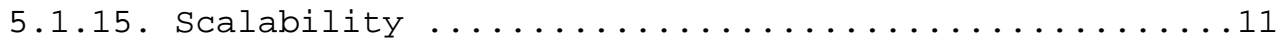

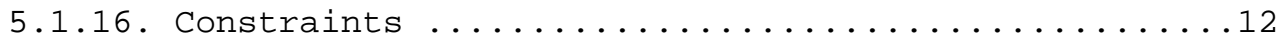

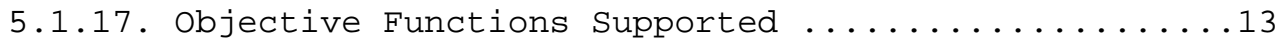

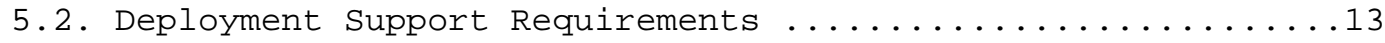

5.2.1. Support for Different Service Provider

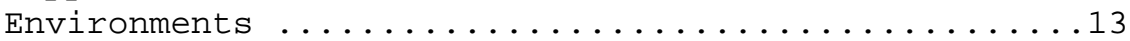

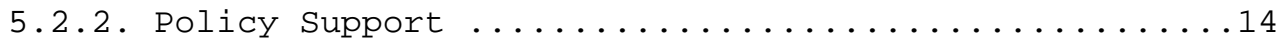

5.3. Aliveness Detection \& Recovery Requirements ...........14

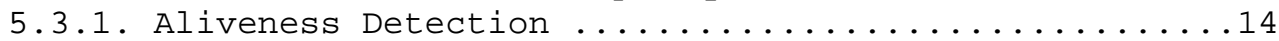

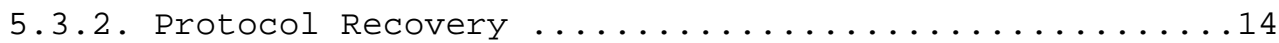

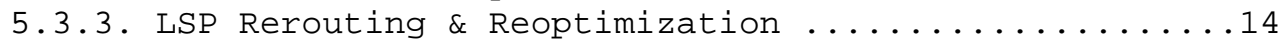

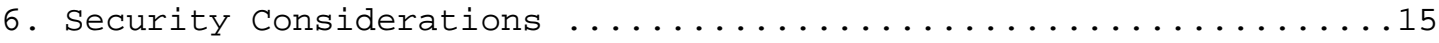

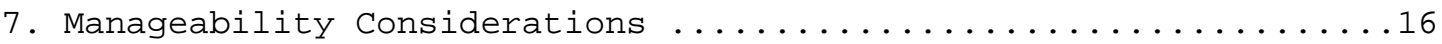

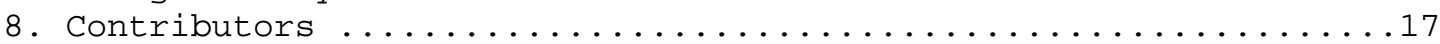

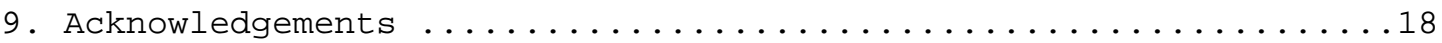

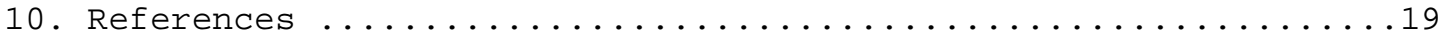

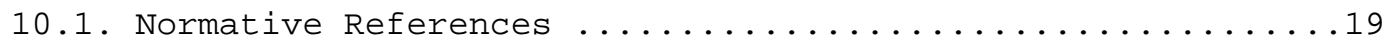

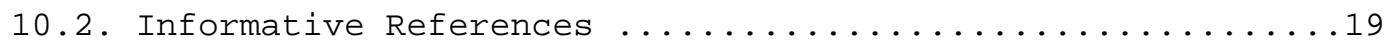

1. Introduction

A Path Computation Element (PCE) [RFC4655] supports requests for path computation issued by a Path Computation Client (PCC), which may be 'composite' (co-located) or 'external' (remote) from a PCE. When the $\mathrm{PCC}$ is external from the $\mathrm{PCE}$, a request/response communication protocol is required to carry the path computation request and return the response. In order for the PCC and PCE to communicate, the PCC must know the location of the PCE; PCE discovery is described in [PCE-DISC-REQ] .

The PCE operates on a network graph in order to compute paths based on the path computation request(s) issued by the PCC(s). The path computation request will include the source and destination of the paths to be computed and a set of constraints to be applied during the computation, and it may also include an objective function. The PCE response includes the computed paths or the reason for a failed computation. 
This document lists a set of generic requirements for the PCE Communication Protocol (PCECP). Application-specific requirements are beyond the scope of this document, and will be addressed in separate documents. For example, application-specific communication protocol requirements are given in [PCECP-INTER-AREA] and [PCECP-INTER-LAYER] for inter-area and inter-layer PCE applications, respectively.

\section{Conventions Used in This Document}

The key words "MUST", "MUST NOT", "REQUIRED", "SHALL", "SHALL NOT", "SHOULD", "SHOULD NOT", "RECOMMENDED", "MAY", "MAY NOT", and "OPTIONAL" in this document are to be interpreted as described in RFC 2119 [RFC2119].

3. Terminology

Domain: Any collection of network elements within a common sphere of address management or path computational responsibility. Examples of domains include Interior Gateway Protocol (IGP) areas, Autonomous Systems (ASS), multiple ASs within a service provider network, or multiple ASs across multiple service provider networks.

GMPLS: Generalized Multi-Protocol Label Switching

LSP: MPLS/GMPLS Label Switched Path

LSR: Label Switch Router

MPLS: Multi-Protocol Label Switching

PCC: Path Computation Client: Any client application requesting a path computation to be performed by the PCE.

PCE: Path Computation Element: An entity (component, application or network node) that is capable of computing a network path or route based on a network graph and applying computational constraints (see further description in [RFC4655]).

TED: Traffic Engineering Database, which contains the topology and resource information of the network or network segment used by a PCE. TE LSP: Traffic Engineering (G) MPLS Label Switched Path. See [RFC4655] for further definitions of terms. 
4. Overview of PCE Communication Protocol (PCECP)

In the PCE model, path computation requests are issued by a PCC to a PCE that may be composite (co-located) or external (remote). If the $\mathrm{PCC}$ and $\mathrm{PCE}$ are not co-located, a request/response communication protocol is required to carry the request and return the response. If the PCC and PCE are co-located, a communication protocol is not required, but implementations may choose to utilize a protocol for exchanges between the components.

In order for a PCC and PCE to communicate, the PCC must know the location of the PCE. This can be configured or discovered. The PCE discovery mechanism is out of scope of this document, but requirements are documented in [PCE-DISC-REQ].

The PCE operates on a network graph built from the TED in order to compute paths. The mechanism by which the TED is populated is out of scope for the PCECP.

A path computation request issued by the PCC includes a specification of the path(s) needed. The information supplied includes, at a minimum, the source and destination for the paths, but may also include a set of further requirements (known as constraints) as described in section 5.

The response from the PCE may be positive in which case it will include the paths that have been computed. If the computation fails or cannot be performed, a negative response is required with an indication of the type of failure.

A request/response protocol is also required for a PCE to communicate path computation requests to another $\mathrm{PCE}$ and for that $\mathrm{PCE}$ to return the path computation response. As described in [RFC4655], there is no reason to assume that two different protocols are needed, and this document assumes that a single protocol will satisfy all requirements for $\mathrm{PCC}-\mathrm{PCE}$ and $\mathrm{PCE}-\mathrm{PCE}$ communication.

[RFC4655] describes four models of PCE: composite, external, multiple PCE path computation, and multiple PCE path computation with interPCE communication. In all cases except the composite PCE model, a $\mathrm{PCECP}$ is required. The requirements defined in this document are applicable to all models described in [RFC4655]. 


\section{PCE Communication Protocol Generic Requirements}

5.1. Basic Protocol Requirements

5.1.1. Commonality of PCC-PCE and PCE-PCE Communication

A single protocol MUST be defined for PCC-PCE and PCE-PCE communication. A PCE requesting a path from another PCE can be considered a PCC, and in the remainder of this document we refer to all communications as $\mathrm{PCC}-\mathrm{PCE}$ regardless of whether they are $\mathrm{PCC}-\mathrm{PCE}$ or $\mathrm{PCE}-\mathrm{PCE}$.

\subsubsection{Client-Server Communication}

PCC-PCE communication is by nature client-server based. The PCECP MUST allow a PCC to send a request message to a PCE to request path computation, and for a PCE to reply with a response message to the requesting $\mathrm{PCC}$ once the path has been computed.

In addition to this request-response mode, there are cases where there is unsolicited communication from the PCE to the PCC (see Section 5.1.11).

5.1.3. Transport

The PCECP SHOULD utilize an existing transport protocol that supports congestion control. This transport protocol may also be used to satisfy some requirements in other sections of this document, such as reliability. The PCECP SHOULD be defined for one transport protocol only in order to ensure interoperability. The transport protocol MUST NOT limit the size of the message used by the PCECP.

\subsubsection{Path Computation Requests}

The path computation request message MUST include at least the source and destination. Note that the path computation request is for an LSP or LSP segment, and the source and destination supplied are the start and end of the computation being requested (i.e., of the LSP segment). 
The path computation request message MUST support the inclusion of a set of one or more path constraints, including but not limited to the requested bandwidth or resources (hops, affinities, etc.) to include/exclude. For example, a PCC may request the PCE to exclude points of failure in the computation of a new path if an LSP setup fails. The actual inclusion of constraints is a choice for the PCC issuing the request. A list of core constraints that must be supported by the PCECP is supplied in Section 5.1.16. Specification of constraints MUST be future-proofed as described in section 5.1.14.

The requester MUST be allowed to select from or prefer an advertised list or minimal subset of standard objective functions and functional options. An objective function is used by the PCE to process constraints to a path computation request when it computes a path in order to select the "best" candidate paths (e.g., minimum hop path), and corresponds to the optimization criteria used for the computation of one path, or the synchronized computation of a set of paths. In the case of unsynchronized path computation, this can be, for example, the path cost or the residual bandwidth on the most loaded path link. In the case of synchronized path computation, this can be, for example, the global bandwidth consumption or the residual bandwidth on the most loaded network link.

A list of core objective functions that MUST be supported by the PCECP is supplied in Section 5.1.17. Specification of objective functions MUST be future-proofed as described in section 5.1.14.

The requester SHOULD also be able to select a vendor-specific or experimental objective function or functional option. Furthermore, the requester MUST be allowed to customize the function/options in use. That is, individual objective functions will often have parameters to be set in the request from PCC to PCE. Support for the specification of objective functions and objective parameters is required in the protocol extensibility specified in section 5.1.14.

A request message MAY include TE parameters carried by the MPLS/GMPLS LSP setup signaling protocol. Also, it MUST be possible for the PCE to apply additional objective functions. This might include policybased routing path computation for load balancing instructed by the management plane.

Shortest path selection may rely either on the TE metric or on the IGP metric [METRIC]. Hence the PCECP request message MUST allow the PCC to indicate the metric type (IGP or TE) to be used for shortest path selection. Note that other metric types may be specified in the future. 
There may be cases where a single path cannot fit a given bandwidth request, while a set of paths could be combined to fit the request. Such path combination to serve a given request is called loadbalancing. The request message MUST allow the PCC to indicate if load-balancing is allowed. It MUST also include the maximum number of paths in a load-balancing path group, and the minimum path bandwidth in a load-balancing path group. The request message MUST allow specification of the degree of disjointness of the members of the load-balancing group.

\subsubsection{Path Computation Responses}

The path computation response message MUST allow the PCE to return various elements including, at least, the computed path(s).

The protocol MUST be capable of returning any explicit path that would be acceptable for use for MPLS and GMPLS LSPs once converted to an Explicit Route Object for use in RSVP-TE signaling. In addition, anything that can be expressed in an Explicit Route object MUST be capable of being returned in the computed path. Note that the resultant path(s) may be made up of a set of strict or loose hops, or any combination of strict and loose hops. Moreover, a hop may have the form of a non-simple abstract node. See [RFC3209] for the definition of strict hop, loose hop, and abstract node.

A positive response from the PCE MUST include the paths that have been computed. A positive PCECP computation response MUST support the inclusion of a set of attributes of the computed path, such as the path costs (e.g., cumulative link TE metrics and cumulative link IGP metrics) and the computed bandwidth. The latter is useful when a single path cannot serve the requested bandwidth and load balancing is applied.

When a path satisfying the constraints cannot be found, or if the computation fails or cannot be performed, a negative response MUST be sent. This response MAY include further details of the reason(s) for the failure and MAY include advice about which constraints might be relaxed to be more likely to achieve a positive result.

The PCECP response message MUST support the inclusion of the set of computed paths of a load-balancing path group, as well as their respective bandwidths.

\subsubsection{Cancellation of Pending Requests}

A PCC MUST be able to cancel a pending request using an appropriate message. A PCC that has sent a request to a PCE and no longer needs a response, for instance, because it no longer wants to set up the 
associated service, MUST be able to notify the PCE that it can clear the request (i.e., stop the computation if already started, and clear the context). The PCE may also wish to cancel a pending request because of some congested state.

\subsubsection{Multiple Requests and Responses}

It MUST be possible to send multiple path computation requests within the same request message. Such requests may be correlated (e.g., requesting disjoint paths) or uncorrelated (requesting paths for unrelated services). It MUST be possible to limit by configuration of both PCCs and PCEs the number of requests that can be carried within a single message.

Similarly, it MUST be possible to return multiple computed paths within the same response message, corresponding either to the same request (e.g., multiple suited paths, paths of a load-balancing path group) or to distinct requests, correlated or not, of the same request message or distinct request messages.

It MUST be possible to provide "continuation correlation" where all related requests or computed paths cannot fit within one message and are carried in a sequence of correlated messages.

The PCE MUST inform the PCC of its capabilities. Maximum acceptable message sizes and the maximum number of requests per message supported by a PCE MAY form part of PCE capabilities advertisement [PCE-DISC-REQ] or MAY be exchanged through information messages from the PCE as part of the protocol described here.

It MUST be possible for a PCC to specify, in the request message, the maximum acceptable response message sizes and the maximum number of computed paths per response message it can support.

It MUST be possible to limit the message size by configuration on PCCs and PCEs.

\subsubsection{Reliable Message Exchange}

The PCECP MUST support reliable transmission of PCECP packets. This may form part of the protocol itself or may be achieved by the selection of a suitable transport protocol (see section 5.1.3).

In particular, it MUST allow for the detection and recovery of lost messages to occur quickly and not impede the operation of the PCECP.

In some cases (e.g., after link failure), a large number of PCCs may simultaneously send requests to a PCE, leading to a potential 
saturation of the PCEs. The PCECP MUST support indication of congestion state and rate limitation state. This should enable, for example, a PCE to limit the rate of incoming request messages if the request rate is too high.

The PCECP or its transport protocol MUST provide the following:

- Detection and report of lost or corrupted messages

- Automatic attempts to retransmit lost messages without reference to the application

- Handling of out-of-order messages

- Handling of duplicate messages

- Flow control and back-pressure to enable throttling of requests and responses

- Rapid PCECP communication failure detection

- Distinction between partner failure and communication channel failure after the PCECP communication is recovered

If it is necessary to add functions to PCECP to overcome shortcomings in the chosen transport mechanisms, these functions SHOULD be based on and re-use where possible techniques developed in other protocols to overcome the same shortcomings. Functionality MUST NOT be added to the PCECP where the chosen transport protocol already provides it.

\subsubsection{Secure Message Exchange}

The PCC-PCE communication protocol MUST include provisions to ensure the security of the exchanges between the entities. In particular, it MUST support mechanisms to prevent spoofing (e.g., authentication), snooping (e.g., preservation of confidentiality of information through techniques such as encryption), and Denial of Service (DoS) attacks (e.g., packet filtering, rate limiting, no promiscuous listening). Once a PCC is identified and authenticated, it has the same privileges as all other PCCs.

To ensure confidentiality, the PCECP SHOULD allow local policy to be configured on the PCE to not provide explicit path(s). If a PCC requests an explicit path when this is not allowed, the PCE MUST return an error message to the requesting PCC and the pending path computation request MUST be discarded.

Authorization requirements [RFC3127] include reject capability, reauthorization on demand, support for access rules and filters, and unsolicited disconnect. 
IP addresses are used to identify PCCs and PCEs. Where the PCC-PCE communication takes place entirely within one limited domain, the use of a private address space that is not available to customer systems MAY be used to help protect the information exchange, but other mechanisms MUST also be available.

These functions may be provided by the transport protocol or directly by the PCECP. See section 6 for further discussion of security considerations.

5.1.10. Request Prioritization

The PCECP MUST allow a PCC to specify the priority of a computation request.

Implementation of priority-based activity within a PCE is subject to implementation and local policy. This application processing is out of scope of the PCECP.

\subsubsection{Unsolicited Notifications}

The normal operational mode is for the PCC to make path computation requests to the $\mathrm{PCE}$ and for the $\mathrm{PCE}$ to respond.

The PCECP MUST support unsolicited notifications from PCE to PCC, or $\mathrm{PCC}$ to PCE. This requirement facilitates the unsolicited communication of information and alerts between PCCs and PCEs. As specified in Section 5.1.8, these notification messages must be supported by a reliable transmission protocol. The PCECP MAY also support response messages to the unsolicited notification messages.

\subsubsection{Asynchronous Communication}

The PCC-PCE protocol MUST allow for asynchronous communication. A PCC MUST NOT have to wait for a response to one request before it can make another request.

It MUST also be possible to have the order of responses differ from the order of the corresponding requests. This may occur, for instance, when path request messages have different priorities (see Requirement 5.1.10). A consequent requirement is that path computation responses MUST include a direct correlation to the associated request.

\subsubsection{Communication Overhead Minimization}

The request and response messages SHOULD be designed so that the communication overhead is minimized. In particular, the overhead per 
message SHOULD be minimized, and the number of bytes exchanged to arrive at a computation answer SHOULD be minimized. Other considerations in overhead minimization include the following:

- the number of background messages used by the protocol or its transport protocol to keep alive any session or association between the PCE and PCC

- the processing cost at the PCE (or PCC) associated with request/response messages (as distinct from processing the computation requests themselves)

\subsubsection{Extensibility}

The PCECP MUST provide a way for the introduction of new path computation constraints, diversity types, objective functions, optimization methods and parameters, and so on, without requiring major modifications in the protocol.

For example, the PCECP MUST be extensible to support various PCEbased applications, such as the following:

- intra-area path computation

- inter-area path computation [PCECP-INTER-AREA]

- inter-AS intra provider and inter-AS inter-provider path computation [PCECP-INTER-AS]

- inter-layer path computation [PCECP-INTER-LAYER]

The PCECP MUST support the requirements specified in the application-specific requirements documents. The PCECP MUST also allow extensions as more PCE applications will be introduced in the future.

The PCECP SHOULD also be extensible to support future applications not currently in the scope of the PCE working group, such as, for instance, point-to-multipoint path computations, multi-hop pseudowire path computation, etc.

Note that application specific requirements are out of the scope of this document and will be addressed in separate requirements documents.

\subsubsection{Scalability}

The PCECP MUST scale well, at least as good as linearly, with an increase of any of the following parameters. Minimum order of magnitude estimates of what the PCECP should support are given in parenthesis (note: these are requirements on the PCECP, not on the $\mathrm{PCE})$ : 
- number of PCCs (1000/domain)

- number of PCEs (100/domain)

- number of PCCs communicating with a single PCE (1000)

- number of PCEs communicated to by a single PCC (100)

- number of domains (20)

- number of path request messages (average of $10 / \mathrm{second} / \mathrm{PCE}$ )

- handling bursts of requests (burst of 100/second/PCE within a 10second interval).

Note that path requests can be bundled in path request messages, for example, $10 \mathrm{PCECP}$ request messages/second may correspond to 100 path requests/second.

Bursts of requests may arise, for example, after a network outage when multiple recomputations are requested. The PCECP MUST handle the congestion in a graceful way so that it does not unduly impact the rest of the network, and so that it does not gate the ability of the PCE to perform computation.

5.1.16. Constraints

This section provides a list of generic constraints that MUST be supported by the PCECP. Other constraints may be added to service specific applications as identified by separate application-specific requirements documents. Note that the provisions of section 5.1.14 mean that new constraints can be added to this list without impacting the protocol to a level that requires major protocol changes.

The set of supported generic constraints MUST include at least the following:

- MPLS-TE and GMPLS generic constraints:

- Bandwidth

- Affinities inclusion/exclusion

- Link, Node, Shared Risk Link Group (SRLG) inclusion/exclusion

- Maximum end-to-end IGP metric

- Maximum hop count

- Maximum end-to-end TE metric

- Degree of paths disjointness (Link, Node, SRLG)

- MPLS-TE specific constraints

- Class-type

- Local protection

- Node protection

- Bandwidth protection 
- GMPLS specific constraints

- Switching type, encoding type

- Link protection type

\subsubsection{Objective Functions Supported}

This section provides a list of generic objective functions that MUST be supported by the PCECP. Other objective functions MAY be added to service specific applications as identified by separate applicationspecific requirements documents. Note that the provisions of Section 5.1.14 mean that new objective functions MAY be added to this list without impacting the protocol.

The PCECP MUST support at least the following "unsynchronized" functions :

- Minimum cost path with respect to a specified metric (shortest path)

- Least loaded path

- Maximum available bandwidth path

Also, the PCECP MUST support at least the following "synchronized" objective functions:

- Minimize aggregate bandwidth consumption on all links

- Maximize the residual bandwidth on the most loaded link

- Minimize the cumulative cost of a set of diverse paths

5.2. Deployment Support Requirements

5.2.1. Support for Different Service Provider Environments

The PCECP must at least support the following environments:

- MPLS-TE and GMPLS networks

- Packet and non-packet networks

- Centralized and distributed PCE path computation

- Single and multiple PCE path computation

For example, PCECP is possibly applicable to packet networks (e.g., IP networks), non-packet networks (e.g., time-division multiplexed (TDM) transport), and perhaps to multi-layer GMPLS control plane environments. Definitions of centralized, distributed, single, and multiple PCE path computation can be found in [RFC4655]. 


\subsubsection{Policy Support}

The PCECP MUST allow for the use of policies to accept/reject requests. It MUST include the ability for a PCE to supply sufficient detail when it rejects a request for policy reasons to allow the PCC to determine the reason for rejection or failure. For example, filtering could be required for a PCE that serves one domain (perhaps an AS) such that all requests that come from another domain (AS) are rejected. However, specific policy details are left to applicationspecific PCECP requirements. Actual policies, configuration of policies, and applicability of policies are out of scope.

Note that work on supported policy models and the corresponding requirements/implications is being undertaken as a separate work item in the PCE working group.

PCECP messages MUST be able to carry transparent policy information.

\subsection{Aliveness Detection \& Recovery Requirements}

\subsubsection{Aliveness Detection}

The PCECP MUST allow a PCC/PCE to

- check the liveliness of the PCC-PCE communication,

- rapidly detect $\mathrm{PCC}-\mathrm{PCE}$ communication failure (indifferently to partner failure or connectivity failure), and

- distinguish PCC/PCE node failures from PCC-PCE connectivity failures, after the PCC-PCE communication is recovered.

The aliveness detection mechanism MUST ensure reciprocal knowledge of $\mathrm{PCE}$ and PCC liveness.

\subsubsection{Protocol Recovery}

In the event of the failure of a sender or of the communication channel, the PCECP, upon recovery, MUST support resynchronization of information (e.g., PCE congestion status) and requests between the sender and the receiver; this SHOULD be arranged so as to minimize repeat data transfer.

\subsubsection{LSP Rerouting \& Reoptimization}

If an LSP fails owing to the failure of a link or node that it traverses, a new computation request may be made to a PCE in order to repair the LSP. Since the PCC cannot know that the PCE'S TED has been updated to reflect the failure network information, it is useful to include this information in the new path computation request. 
Also, in order to re-use the resources used by the old LSP, it may be advantageous to indicate the route of the old LSP as part of the new path computation request.

Hence the path computation request message MUST allow an indication of whether the computation is for LSP restoration, and it MUST support the inclusion of the previously computed path as well as the identity of the failed element. Note that the old path might only be useful if the old LSP has not yet been torn down. The PCE MAY choose to take failure indication information carried in a given request into account when handling subsequent requests. This should be driven by local policy decision.

Note that a network failure may impact a large number of LSPs. In this case, a potentially large number of PCCs will simultaneously send requests to the PCE. The PCECP MUST properly handle such overload situations, such as, for instance, through throttling of requests as set forth in section 5.1.8.

The path computation request message MUST support TE LSP path reoptimization and the inclusion of a previously computed path. This will help ensure optimal routing of a reoptimized path, since it will allow the PCE to avoid double bandwidth accounting and help reduce blocking issues.

\section{Security Considerations}

Key management MUST be provided by the PCECP to provide for the authenticity and integrity of PCECP messages. This will allow protecting against PCE or PCC impersonation and also against message content falsification.

The impact of the use of a PCECP MUST be considered in light of the impact that it has on the security of the existing routing and signaling protocols and techniques in use within the network. Intra-domain security is impacted since there is a new interface, protocol, and element in the network. Any host in the network could impersonate a PCC and receive detailed information on network paths. Any host could also impersonate a PCE, both gathering information about the network before passing the request on to a real PCE and spoofing responses. Some protection here depends on the security of the PCE discovery process (see [PCE-DISC-REQ]). An increase in inter-domain information flows may increase the vulnerability to security attacks, and the facilitation of inter-domain paths may increase the impact of these security attacks.

Of particular relevance are the implications for confidentiality inherent in a PCECP for multi-domain networks. It is not necessarily 
the case that a multi-domain PCE solution will compromise security, but solutions MUST examine their impacts in this area.

Applicability statements for particular combinations of signaling, routing, and path computation techniques are expected to contain detailed security sections.

It should be observed that the use of an external PCE introduces additional security issues. Most notable among these are the following:

- Interception of PCE requests or responses

- Impersonation of PCE or PCC

- Dos attacks on PCEs or PCCs

The PCECP MUST address these issues in detail using authentication, encryption, and DoS protection techniques. See also Section 5.1.9.

There are security implications of allowing arbitrary objective functions, as discussed in section 5.1.17, and the PCECP MUST allow mitigating the risk of, for example, a PCC using complex objectives to intentionally drive a PCE into resource exhaustion.

7. Manageability Considerations

Manageability of the PCECP MUST address the following considerations:

- The need for a MIB module for control and monitoring of PCECP

- The need for built-in diagnostic tools to test the operation of the protocol (e.g., partner failure detection, Operations Administration and Maintenance (OAM), etc.)

- Configuration implications for the protocol

PCECP operations MUST be modeled and controlled through appropriate MIB modules. There are enough specific differences between PCCs and PCEs to lead to the need of defining separate MIB modules.

Statistics gathering will form an important part of the operation of the PCECP. The MIB modules MUST provide information that will allow an operator to determine PCECP historical interactions and the success rate of requests. Similarly, it is important for an operator to be able to determine PCECP and PCE load and whether an individual PCC is responsible for a disproportionate amount of the load. It MUST be possible, through use of MIB modules, to record and inspect statistics about the PCECP communications, including issues such as malformed messages, unauthorized messages, and messages discarded owing to congestion. 
The new MIB modules should also be used to provide notifications (traps) when thresholds are crossed or when important events occur. For example, the MIB module may support indication of exceeding the congestion state threshold or rate limitation state.

PCECP techniques must enable a PCC to determine the liveness of a PCE both before it sends a request and in the period between sending a request and receiving a response.

It is also important for a PCE to know about the liveness of PCCs to gain a predictive view of the likely loading of a PCE in the future and to allow a PCE to abandon processing of a received request.

The PCECP MUST support indication of congestion state and rate limitation state, and MAY allow the operator to control such a function.

8. Contributors

This document is the result of the PCE Working Group PCECP requirements design team joint effort. In addition to the authors/editors listed in the "Authors' Addresses" section, the following are the design team members who contributed to the document:

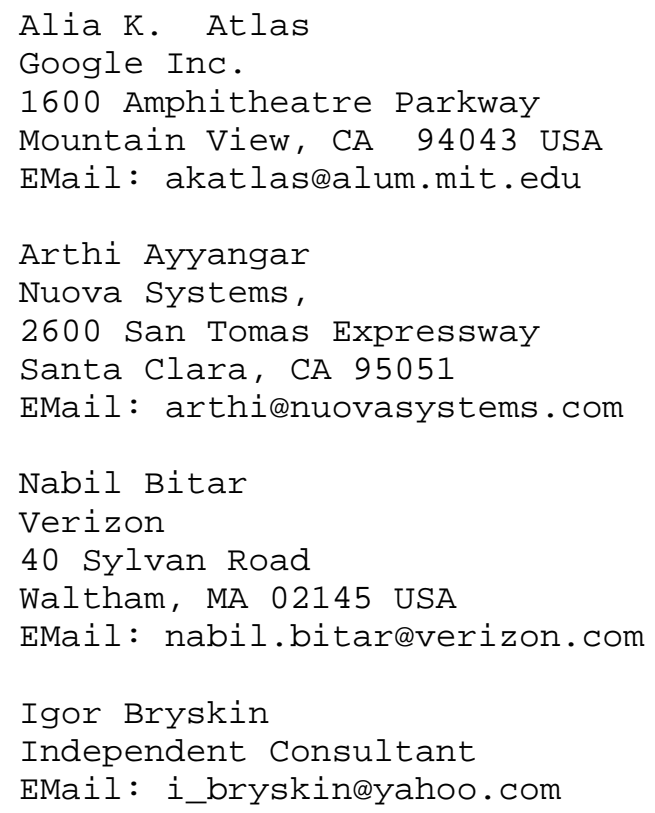




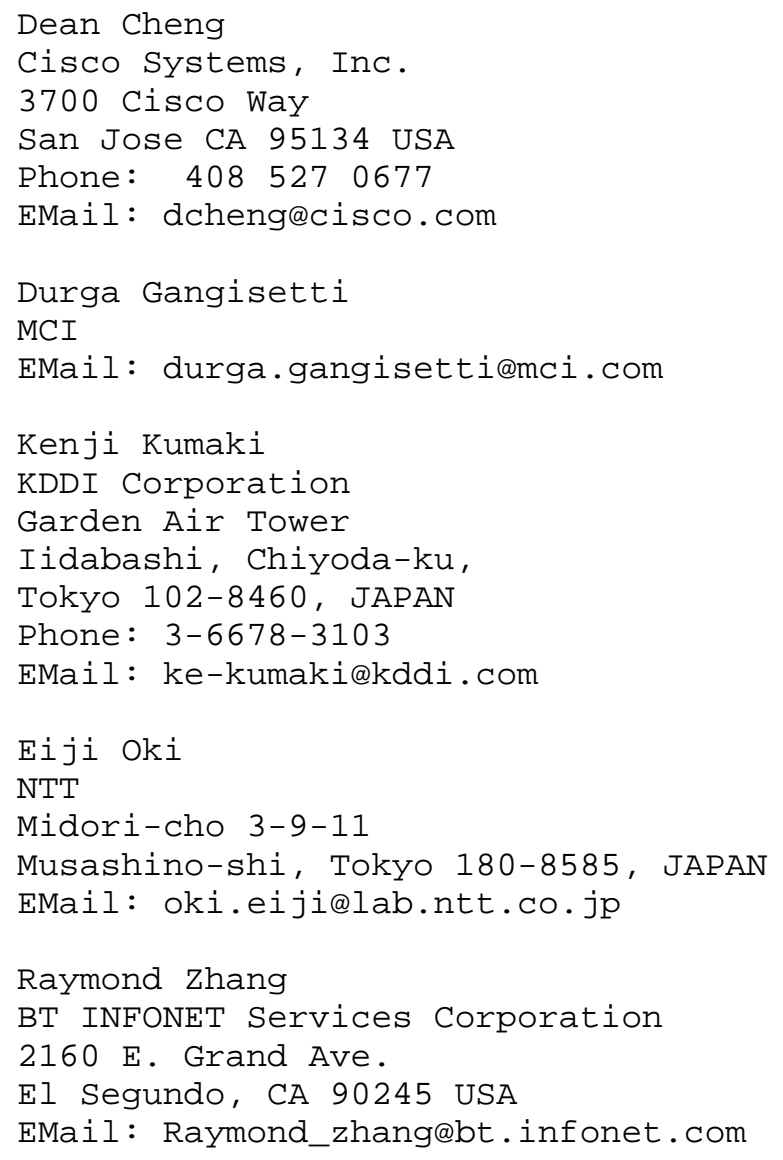

9. Acknowledgements

The authors would like to extend their warmest thanks to (in alphabetical order) Lou Berger, Ross Callon, Adrian Farrel, Thomas Morin, Dimitri Papadimitriou, Robert Sparks, and J.P. Vasseur for their review and suggestions. 
10. References

10.1. Normative References

[RFC2119] Bradner, S., "Key words for use in RFCs to Indicate Requirement Levels", BCP 14, RFC 2119, March 1997.

[RFC4655]

Farrel, A., Vasseur, J.-P., and J. Ash, "A Path Computation Element (PCE)-Based Architecture", RFC 4655, August 2006.

10.2. Informative References

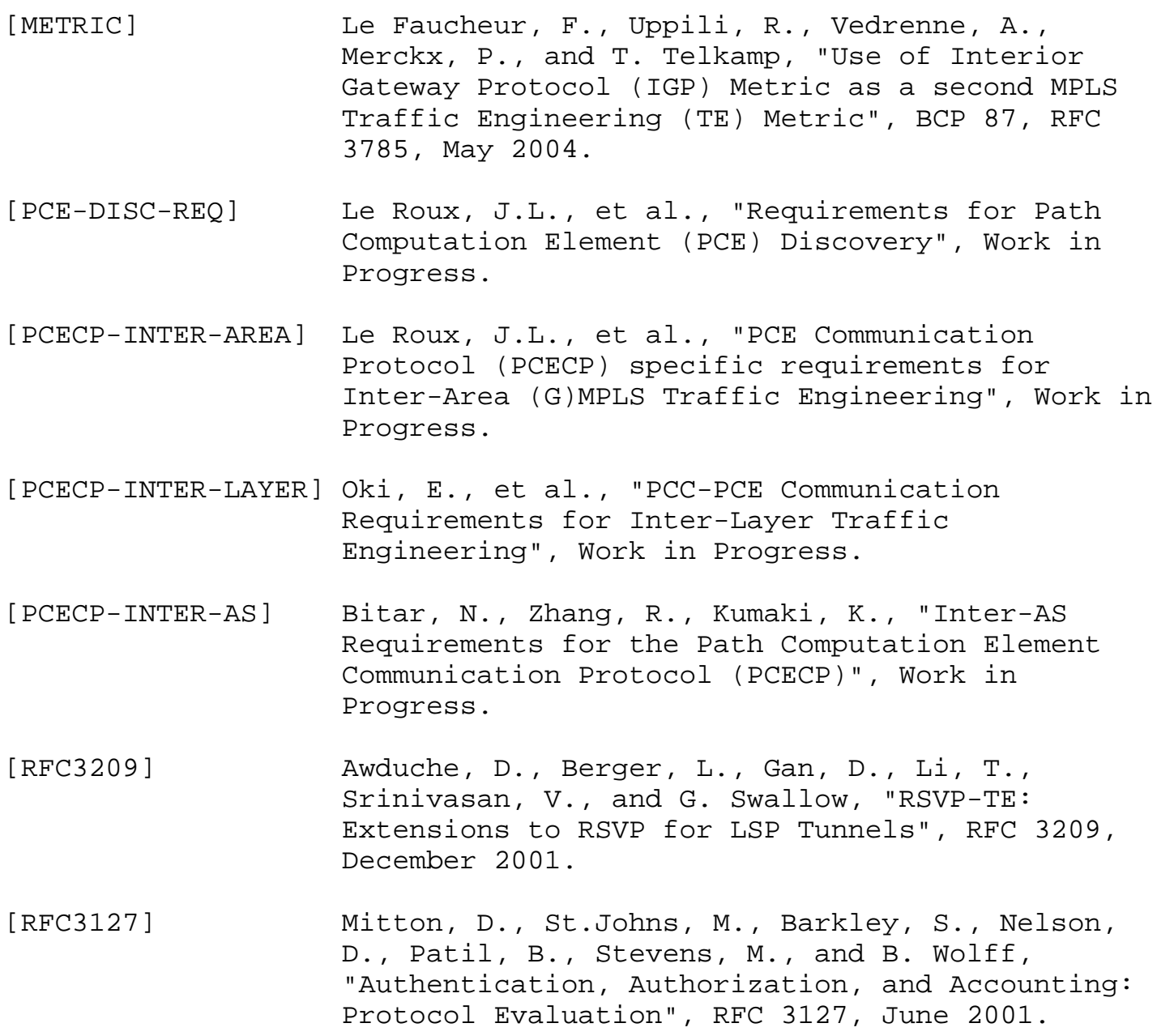

[RFC3209]

Awduche, D., Berger, L., Gan, D., Li, T., Srinivasan, V., and G. Swallow, "RSVP-TE: Extensions to RSVP for LSP Tunnels", RFC 3209, December 2001 .

$[\mathrm{RFC} 3127]$

Mitton, D., St.Johns, M., Barkley, S., Nelson, D., Patil, B., Stevens, M., and B. Wolff, "Authentication, Authorization, and Accounting: Protocol Evaluation", RFC 3127, June 2001. 


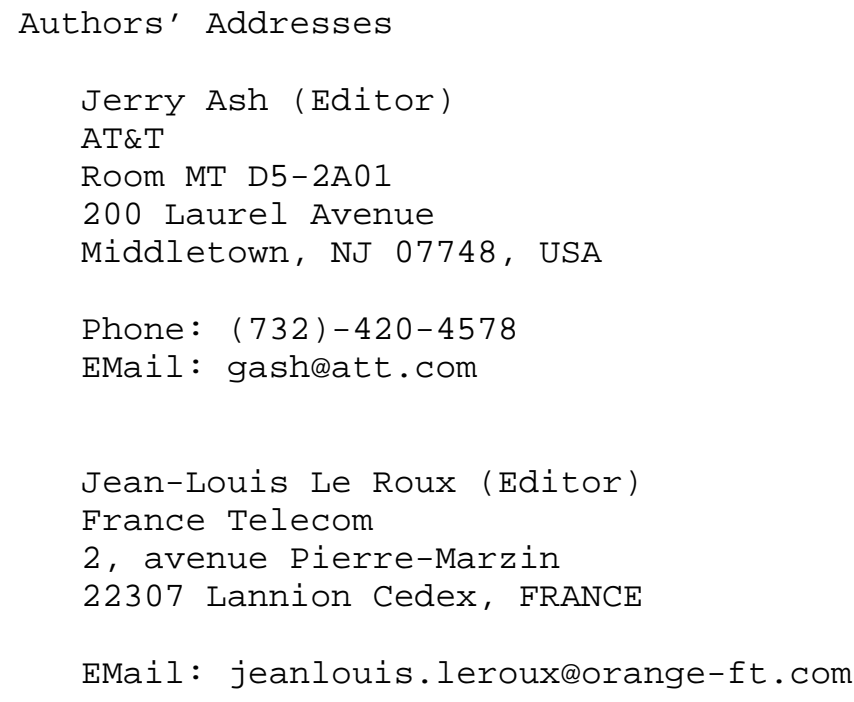


Full Copyright statement

Copyright (C) The Internet Society (2006).

This document is subject to the rights, licenses and restrictions contained in BCP 78, and except as set forth therein, the authors retain all their rights.

This document and the information contained herein are provided on an "AS IS" basis and THE CONTRIBUTOR, THE ORGANIZATION HE/SHE REPRESENTS OR IS SPONSORED BY (IF ANY), THE INTERNET SOCIETY AND THE INTERNET ENGINEERING TASK FORCE DISCLAIM ALL WARRANTIES, EXPRESS OR IMPLIED, INCLUDING BUT NOT LIMITED TO ANY WARRANTY THAT THE USE OF THE INFORMATION HEREIN WILL NOT INFRINGE ANY RIGHTS OR ANY IMPLIED WARRANTIES OF MERCHANTABILITY OR FITNESS FOR A PARTICULAR PURPOSE.

Intellectual Property

The IETF takes no position regarding the validity or scope of any Intellectual Property Rights or other rights that might be claimed to pertain to the implementation or use of the technology described in this document or the extent to which any license under such rights might or might not be available; nor does it represent that it has made any independent effort to identify any such rights. Information on the procedures with respect to rights in RFC documents can be found in BCP 78 and BCP 79 .

Copies of IPR disclosures made to the IETF Secretariat and any assurances of licenses to be made available, or the result of an attempt made to obtain a general license or permission for the use of such proprietary rights by implementers or users of this specification can be obtained from the IETF on-line IPR repository at http://www.ietf.org/ipr.

The IETF invites any interested party to bring to its attention any copyrights, patents or patent applications, or other proprietary rights that may cover technology that may be required to implement this standard. Please address the information to the IETF at ietf-ipreietf.org.

Acknowledgement

Funding for the RFC Editor function is provided by the IETF Administrative Support Activity (IASA). 